\title{
HIGIENE BUCAL EM PRÉ-ESCOLARES PERTENCENTES A UM CENTRO DE REFERÊNCIA DE EDUCAÇÃO INFANTIL EM JOÃO PESSOA-PB
}

\author{
Alayde Pinto Veras, \\ Elaine Cristina Velêz Rodrigues, \\ Maria Karoline de Brito Alves \\ Margarida Maria Pontes de Carvalho \\ Jainara Maria Soares Ferreira
}

\begin{abstract}
RESUMO
A cárie dentária e a gengivite ainda são consideradas um problema de saúde pública no Brasil na faixa etária pré-escolar. Deste forma, a higiene bucal adequada, por métodos mecânicos, com a desorganização constante e eficaz do biofilme, é necessária para prevenção destas doenças bucais que são frequentes em idades precoces. Neste sentido, o objetivo deste trabalho foi avaliar a condição de higiene bucal de crianças na faixa etária de 2 a 5 anos, matriculadas em uma CREI da cidade de João Pessoa (PB). Trata-se de um estudo quantitativo, observacional, transversal e descritivo, realizado em uma CREl, situada no bairro do Valentina. A amostra foi censitária havendo adesão de 77 responsáveis neste estudo $(64,2 \%)$. Os dados foram obtidos por meio de um questionário estruturado aos responsáveis pelas crianças, com perguntas objetivas, relacionadas ao acesso e uso de métodos químicos e mecânicos de controle do biofilme dental, em pré-escolares e na forma de estatística descritiva, através de números absolutos e percentuais. Verificou-se que $93,5 \%$ das crianças utilizavam escovas infantis e $70,1 \%$ creme dental fluoretado, sendo $81,8 \%$ os pais/responsáveis encarregados pela escovação dentária das crianças. Além disso, $27,3 \%$ das crianças pesquisadas escovavam os dentes com frequência igual ou superior a $3 \mathrm{x}$ ao dia, $46,8 \%$ usavam quantidade intermediária de creme dental, durante a escovação, e 11,7\% das crianças ingeriam dentifrício em momentos diferentes da escovação. Houve baixa frequência com relação ao uso do fio dental por parte das crianças (11,7\%), sendo os pais/responsáveis os detentores desta tarefa $(7,8 \%)$, com frequência de três vezes ou mais por semana $(5,2 \%)$. Os pais relataram o uso de bochechos em $22,1 \%$ das crianças. Conclui-se que a maioria dos participantes apresentou boas práticas quanto ao uso destes artigos, porém condutas insatisfatórias foram relatos frequentes com relação ao uso do fio dental e de bochechos.
\end{abstract}

PALAVRAS-CHAVE

Higiene Bucal. Pré-Escolares. Educação Infantil.

\section{INTRODUÇÃO}

Apesar da diminuição da prevalência de cárie dentária e do aumento do número de pessoas, que nunca tiveram experiência de cárie, ter sido observado em diversos países, a doença ainda é um importante problema de saúde pública, e as sequelas dela decorrente, como a perda dentária, são consideradas preocupantes, sendo necessário manter monitoramento constante sobre a doença, principalmente em populações de baixa renda e com menor possibili- 
dade de acesso aos serviços de assistência e prevenção 1 .

Estudos apontam variação da prevalência da cárie dentária entre $16,4 \%$ a $86,7 \%$ em pré-escolares, em João Pessoa (PB). Segundo o último levantamento epidemiológico nacional, realizado em 2010, as crianças de 5 anos apresentam prevalência de 60,1\% desta patologia2,4.

Acredita-se que o grande desafio da Odontologia atual é atuar educativamente junto à população infantil, provendo-a de informações necessárias ao desenvolvimento de hábitos para manter a saúde e prevenir as doenças bucais 5 .

Os primeiros anos de vida das crianças são ideais para instituição de hábitos ou práticas que levam à preservação e à manutenção da saúde bucal, com o auxílio dos familiares, professores e cuidadores ${ }^{6}$.

Segundo a Associação Dentária Americana ( $A D A)^{7}$, a escova ideal deve apresentar certas características, tais como: tufos de cerdas com o mesmo comprimento, cabeça e haste situada no mesmo eixo, leveza e facilidade de limpeza, impermeabilidade à umidade, cerdas de nylon, cabeça pequena, baixo custo, durabilidade e eficiência no controle de placa 8 . Em 1934, Fones 9 apresentou uma técnica de escovação que ainda hoje é usada para crianças, em idade pré-escolar, e consiste em utilizar movimentos circulares amplos, com a escova colocada perpendicularmente em relação ao longo eixo dos dentes com as arcadas fechadas. Todavia, a melhor técnica deve ser considerada a que o paciente melhor se adapte.

Com relação ao uso de dentifrícios em bebês, a Associação Brasileira de Odontopediatria ${ }^{10}$ afirma que, a partir do momento em que os dentes nascem, já podem receber os benefícios do contato com o flúor. Evidências científi- cas sugerem que a ação preventiva da escovação pode ser maximizada se os seguintes princípios forem seguidos: assim que os dentes decíduos irrompem na boca, a recomendação é escová-los, no mínimo, duas vezes ao dia com um creme dental fluoretado, contendo nada menos do que 1.000ppm de flúor, devendo essas duas vezes se dividirem em a última coisa a ser feita à noite e pelo menos uma outra vez a cada dia. É uma boa prática que crianças menores de três anos usem apenas uma mancha de creme dental (uma fina camada de pasta cobrindo menos de três quartos da escova) e não deve ser permitido comer ou lamber creme dental do tubo. Aconselha-se que a escovação aconteça sob a supervisão de adultos e respeitando essa quantidade ${ }^{11}$.

Apenas a escova dental pode não ser suficiente para realizar uma efetiva higiene bucal na região interproximal e pode ser suplementada por outros métodos mecânicos, a exemplo do fio dental. Os fios dentais podem ser encontrados de diferentes formas no mercado, encerados ou não encerados, finos ou grossos, com ou sem sabor, com ou sem flúor. Não existem evidências científicas sobre a superioridade de um em relação aos demais ${ }^{12}$. O fio dental pode ser usado por meio do método do "fio livre" ou da "alça". Vale ressaltar que crianças, em idade pré-escolar, tinham dificuldade motora de realizar seu uso, sendo necessários o treinamento e supervisão dos pais. Com relação ao uso de bochechos na idade pré-escolar, é contraindicado seu uso nesta faixa etária, devido sua pouca habilidade motora e risco de ingestão de grande quantidade do produto ${ }^{13}$.

Neste sentido, os objetivos deste trabalho foram avaliar a condição de higiene bucal de crianças, na faixa etária de 2 a 5 anos, matriculadas em uma CREI da cidade de João Pessoa (PB). 


\section{MATERIAIS E MÉTODOS}

Trata-se de uma pesquisa de estudo quantitativo, observacional, transversal e descritivo. O estudo foi realizado em uma CREI situada no bairro do Valentina. O universo foi constituído por responsáveis de crianças na faixa etária de 2 a 5 anos de idade $(\mathrm{N}=120)$, matriculadas na referida CREI. A amostra é do tipo censitária seguindo os critérios de elegibilidade estabelecidos. Neste sentido, houve adesão de 77 responsáveis neste estudo (64,2\%).

Após aprovação do CEP local (Protocolo CEP: 99/2017 e CAAE: 71006317.5.0000.5179.), explicações aos pesquisados sobre o estudo e obtenção da assinatura do Termo de Consentimento Livre e Esclarecido (TCLE) dos pesquisados, respeitando a resolução CNS/MS 466/2012, foi aplicado um questionário estruturado aos responsáveis pelas crianças com perguntas objetivas relacionadas ao acesso e uso de métodos químicos e mecânicos de controle do biofilme dental em pré-escolares.

Os dados coletados foram analisados pelo programa estatístico SPSS V.20.0 e trabalhados na forma de estatística descritiva, por meio de números absolutos e percentuais.

\section{RESULTADOS E DISCUSSÃO}

No presente estudo, com relação à faixa etária das crianças estudadas, observou-se que $11,7 \%$ ( $n=9)$ compreendia a idade

de 2 anos, 24,2\% ( $n=19)$ estava com 3 anos, $18,2 \%(n=14)$ tinham 4 anos e 45,5\% ( $n=35), 5$ anos, conforme elenca o gráfico 1.

Gráfico 1 - Distribuição por idade das crianças participantes. João Pessoa, PB, 2017.

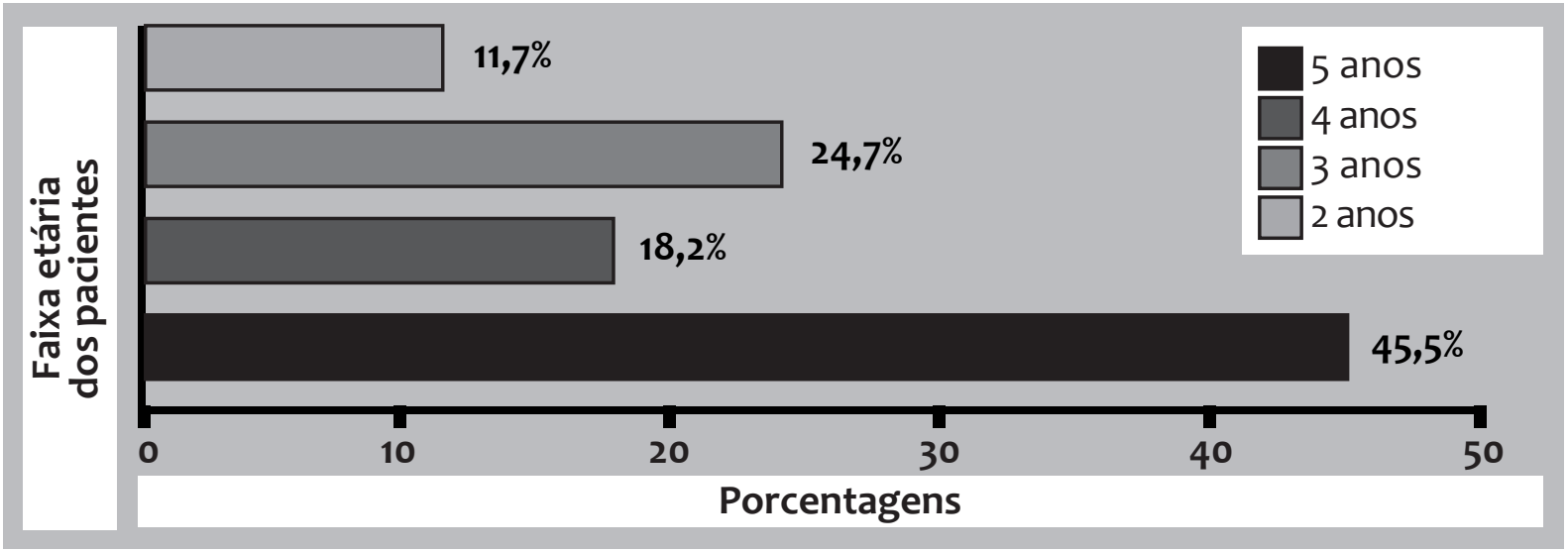

O gráfico 2 exibe o gênero dos participantes da pesquisa. O gênero femi- nino $(55,8 \%, \mathrm{n}=43)$ foi predominante em relação ao masculino $(44,2 \%, n=34)$.

Gráfico 2 - Distribuição por gênero das crianças participantes. João Pessoa, PB, 2017.

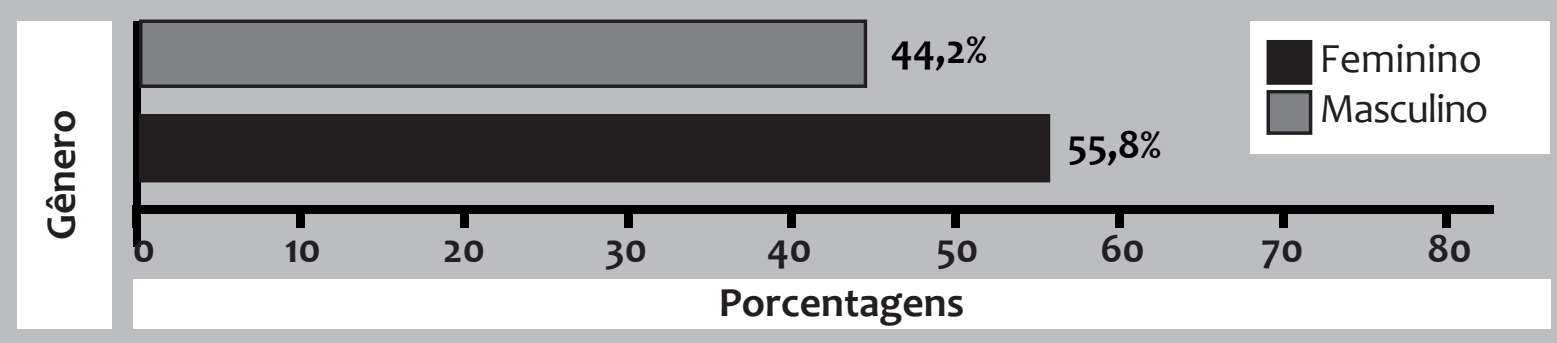


A tabela 1 informa a respeito do acesso e uso dos métodos mecânicos e químicos para controle do biofilme dental nos pré-escolares pesquisados.

Tabela 1 - Associação de achados Endoscópicos no Esôfago nas EDA avaliadas

\begin{tabular}{|c|c|c|c|}
\hline Perguntas & Respostas & $\mathbf{n}$ & $\%$ \\
\hline \multirow{3}{*}{ 1. A criança tem escova de dente? } & Sim & 76 & 98,7 \\
\hline & Não & - & - \\
\hline & Não sabe & 1 & 1,3 \\
\hline \multirow[t]{3}{*}{ 1.1. Se positivo, é de adulto ou de criança? } & De adulto & 3 & 3,9 \\
\hline & De criança & 72 & 93,5 \\
\hline & Não sabe & 1 & 1,3 \\
\hline \multirow[t]{3}{*}{ 2. A criança tem pasta de dente? } & Sim & 69 & 89,6 \\
\hline & Não & 6 & 7,8 \\
\hline & Não sabe & 2 & 2,6 \\
\hline \multirow[t]{3}{*}{ 2.1. Se positivo, é com ou sem flúor? } & Com flúor & 54 & 70,1 \\
\hline & Sem flúr & 11 & 14,3 \\
\hline & Não sabe & 10 & 13,0 \\
\hline \multirow[t]{4}{*}{ 3. Quantas vezes a criança escova os dentes ao dia? } & $1 \mathrm{x}$ & 4 & 5,2 \\
\hline & $2 x$ & 49 & 63,6 \\
\hline & 3 ou mais $x$ & 21 & 27,3 \\
\hline & Não sabe & 3 & 3,9 \\
\hline \multirow[t]{4}{*}{ 4. Quem realiza as escovações da criança? } & criança & 11 & 14,3 \\
\hline & pais/responsáveis & 63 & 81,8 \\
\hline & Outro & 1 & 1,3 \\
\hline & Não sabe & 2 & 2,6 \\
\hline \multirow[t]{4}{*}{ 5. Qual quantidade de pasta de dente colocada na escova? } & Pequena & 26 & 33,8 \\
\hline & Intermediária & 36 & 46,8 \\
\hline & Grande & 7 & 9,1 \\
\hline & Não sabe & 8 & 10,4 \\
\hline \multirow{3}{*}{$\begin{array}{l}\text { 6. A criança tem hábito de ingerir pasta de dente em } \\
\text { outros momentos, além da escovação? }\end{array}$} & $\operatorname{Sim}$ & 9 & 11,7 \\
\hline & Não & 65 & 84,4 \\
\hline & Não sabe & 3 & 3,9 \\
\hline \multirow[t]{3}{*}{ 7. A criança faz uso de fio dental? } & $\operatorname{Sim}$ & 9 & 11,7 \\
\hline & Não & 63 & 81,8 \\
\hline & Não sabe & 5 & 6,5 \\
\hline \multirow[t]{4}{*}{ 7.1. Se positivo, quem passa o fio? } & Criança & 1 & 1,3 \\
\hline & Pais/responsáveis & 6 & 7,8 \\
\hline & Outro & - & - \\
\hline & Não sabe & 2 & 2,6 \\
\hline \multirow[t]{4}{*}{ 7.2. Se positivo, qual frequência semanal? } & $1 \mathrm{x}$ & 1 & 1,3 \\
\hline & $2 x$ & 1 & 1,3 \\
\hline & 3 ou mais $x$ & 4 & 5,2 \\
\hline & Não sabe & 3 & 3,9 \\
\hline \multirow[t]{3}{*}{ 8. A criança faz uso de bochechos? } & Sim & 17 & 22,1 \\
\hline & Não & 57 & 74 \\
\hline & Não sabe & 3 & 3,9 \\
\hline
\end{tabular}


Um número expressivo de crianças tinha acesso à escova dental (98,7\%) e ao creme dental $(89,6 \%)$ Com relação ao tipo da escova e creme dental que as crianças utilizavam, 93,5\% utilizavam escovas infantis e $70,1 \%$ creme dental fluoretado. No tocante a estas informações, Bengtson e outros autores $^{15}$ e Corrêa e outros autores ${ }^{22}$ preconizam o início do uso da escova de dente aos 18 meses. A escova infantil é indicada, desde que tenha cabeça pequena, cerda macia e cabo reto ${ }^{15,22}$. Porém, o uso de dentifrícios com flúor em crianças de baixa idade deve ser usado com cautela, devido a dificuldade de coordenação motora nesta etapa de vida, sabor agradável e concentração padrão de flúor superior a 1000 ppm disponíveis no mercado brasileiro. Albuquerque e outros autores ${ }^{17}$ observaram que o tipo de dentifrício mais utilizado nas instituições públicas foi o adulto $(48,5 \%)$ e nas particulares, o infantil (83,6\%).

Vale ressaltar que, segundo o Ministério da Saúde ${ }^{14}$, o dentifrício fluoretado é considerado um dos mais racionais de prevenção da cárie, pois alia a remoção do biofilme dentário à exposição constante ao flúor. Sua utilização tem sido considerada responsável pela diminuição nos índices de cárie observados hoje em todo mundo, mesmo em países ou regiões que não têm água fluoretada. $O$ dentifrício não fluoretado é uma medida que não encontra respaldo científico. Além disso, a menor concentração de flúor também não resolveria o problema do risco de fluorose em termos de indivíduos, sendo que, por isso, seu uso não é recomendado. Ressalta-se que usar uma pequena quantidade de dentifrício é a medida mais importante para reduzir o risco de fluorose ${ }^{18}$.

Apenas $27,3 \%$ das crianças pesquisadas escovavam os dentes com frequência igual ou superior a $3 \mathrm{x}$ ao dia, o que discorda das recomendações de Stuani e outros autores $^{19}$ que indicam a frequência ideal de escovação, após a dentição decídua completa, três vezes ao dia. O prazer no ato da higiene bucal pode ser fruto do processo de educação em saúde bucal em idades precoces 23 .

Os pais/responsáveis, junto com as crianças, eram responsáveis pela escovação dentária na maior parte dos casos (81,8\%). Este achado concorda com Cangussu e outros autores ${ }^{20}$ ao referir a importância da supervisão dos pais no momento da escovação em crianças menores de 7 anos.

Os indivíduos pesquisados afirmam, por meio de representação gráfica, que usavam uma quantidade intermediária de creme dental (46,8\%). De forma semelhante, Albuquerque e outros autores ${ }^{17}$ observaram que em instituições particulares e públicas, 57,8\% e 50,9\% das crianças utilizam quantidade média de dentifrício, respectivamente. Além disso, os responsáveis relataram que $11,7 \%$ das crianças ingeriam dentifrício em momentos diferentes da escovação. Nesta perspectiva, Moraes e outros autores 24 apontam a necessidade de se instruir pais e cuidadores de crianças a utilizarem uma quantidade pequena de dentifrício $(<0,3 g)$. Concentração de flúor reduzida por pré-escolares constitui em boa alternativa para se minimizar a ingestão de flúor e risco de fluorose dentária.

Foi observada baixa frequência com relação ao uso do fio dental por parte das crianças (11,7\%), sendo os pais/responsáveis os detentores desta tarefa $(7,8 \%)$, com frequência de três vezes ou mais por semana $(5,2 \%)$. Este fato provavelmente pode estar associado ao baixo poder aquisitivo, ou falta de informação dos participantes. Quando comparado à escova de dente, aquele é um produto mais caro e menos difundido, sendo pouco incorporado ao cotidiano da população de baixa renda ${ }^{21}$.

Com relação ao uso de bochechos, os pais relataram que $22,1 \%$ das crianças faziam uso deste produto, o que discorda das recomendações da literatura ao indicar o uso do enxaguatório bucal apenas para crianças em idade escolar ${ }^{24}$. 


\title{
CONSIDERAÇÕES FINAIS
}

Diante do exposto, é lícito concluir que o uso de escova dental infantil e dentifrício fluoretado é a forma mais utilizada para higiene. A maioria dos participantes apresentou boas práticas, quanto ao uso destes artigos, porém condutas insatisfatórias foram relatos frequentes com relação ao uso do fio dental e de bochechos. Os pais ou responsáveis, em sua maioria, supervisionavam estes procedimentos.

\section{ORAL HYGIENE IN PRESCHOOL CHILDREN AT AN EARLY CHILDHOOD EDUCATION CENTER IN JOÃO PESSOA, PARAÍBA STATE}

\begin{abstract}
Dental caries and gingivitis are still considered a public health problem in Brazil at the pre-school age. So, a proper oral hygiene by mechanical methods with the constant and effective biofilm disorganization is necessary to prevent these oral diseases that are frequent at early ages. Therefore, this study objective was to evaluate the oral hygiene condition of children between 2 and 5 years old enrolled on a Early Childhood Education Center in João Pessoa (PB). It was a quantitative, observational, cross-sectional and descriptive study carried out on ECEC located in the Valentina neighborhood. At this study, the sample was census-based, with 77 respondents (64.2\%). The data were obtained through a structured questionnaire answered by those responsible for children, with objective questions related to the access and use of chemical and mechanical methods of dental biofilm control in preschool children, and in descriptive statistics, using absolute and percentage numbers. It was found that $93.5 \%$ of the children used toothbrushes and $70.1 \%$ used fluoride toothpaste, $81.8 \%$ of which were the parents/guardians responsible for tooth brushing. Furthermore, $27.3 \%$ of the surveyed children brushed their teeth three or more times a day, $46.8 \%$ used an average amount of toothpaste during toothbrushing and $11.7 \%$ of the children drank toothpaste at different times of brushing process. There was a low frequency of flossing by the children (11.7\%), the parents/guardians being responsible for this task (7.8\%), with frequency of three times or more per week (5.2\%). The parents reported the use of mouthwash in $22.1 \%$ of the children. We can conclude that most of the participants presented good practices regarding the use of these instruments, but unsatisfactory conducts were frequent reports regarding the use of dental floss and mouthwash.
\end{abstract}

\section{KEYWORDS}

Oral Hygiene. Preschool children. Childhood Education.

\section{REFERÊNCIAS}

1 Santos MO, Casotti CA, Queiroz APDG, Carneiro JAO, Uermura TF. Conhecimento e percepção sobre saúde bucal de professores e pré-escolares de um município baiano. RFO. 2015; 20 (2):172-8.

2 Graville-gracia AF, Ferreira JMS, Barbosa AMF, Vieira I. et al. Cárie, gengivite e higiene bucal em pré-escolares. Rev. Gaúch. Odontol. 2010; .58(4): 469-73.

3 Limera AB, Lima FRSB, Franca C, Colares
V. et al. Prevalência de cáries em crianças e cuidadores de uma creche em Recife/PE. Odontol. Clín.-Cient. 2010; 9 (4):325-9.

4 Avellar-silva ABV, Oliveira LMC, Silveira RG, Miasato JM et al. Prevalência de cárie dentária em pré-escolares de uma escola particular em uma região rural do Estado do Rio de Janeiro. Revista Brasileira de Pesquisa em Saúde. 2012; 14(1): 49-56. 
Paiva SM. Escola: um espaço importante de informação em saúde bucal para a população infantil. PGR-Pós-Grad Rev Fac Odontol São José dos Campos. 2001; 4 (3): 43-51.

6 Franchin V, Basting RT, Mussi AA, Flório FM. A importância do professor como agente multiplicador de Saúde Bucal. Rev Abeno 2006; 6(2):102-8.

7 American Dental Association, American Academy of Periodontology. ADA and AAP introduce dentist to new time saving periodontal evaluation system. Va Dent J. 1992;69(4):16-17.

8 Zaze ACSF, Oliveira ER de, Melão M de JA da S, Alves E. Eficácia de diferentes tipos de escovas dentais na remoção do biofilme bucal. Arq. Cienc. Saúde UNIPAR. 2016; 20 (2):101-9.

9 FONES, C.A. Mouth Hygiene. Lea \& Febiger. 1934; 294-306.

10 Associação Brasileira de Odontopediatria. Flúor - a partir de qual idade utilizar. São Paulo, SP: A Associação; 2017.

11 Wong MCM, Glenny AM, Tsang BWK, Lo ECM. et al. Topical fluoride as a cause of dental fluorosis in children. Evid.-Based Child Health. 2011; 6: 388-439.

12 Bass CC. The optimum characteristics of dental floss for personal oral hygiene. Dental Items of Interrest. 1948; 70: 921-34.

13 São Paulo. Secretaria de Estado de Saúde. Recomendações sobre uso de produtos fluorados no âmbito do SUS/SP em função do risco de cárie dentária. São PauIo, SP: A Secretaria; 2000.

14 Brasil. Ministério da Saúde. Secretaria de Atenção à Saúde. Departamento de Atenção Básica. Coordenação Geral de Saúde Bucal. Projeto SB Brasil 2010: Pesquisa Nacional de Saúde Bucal Resultados Principais. Brasília, DF: O Ministério; 2011.

15 Bengtson NG, Bengtson AL. Bengtson CRG, Pinheiro SL, Cichello LRD. Educação e higiene bucal de bebês: dispositivos e escovas dentais do mercado brasileiro. J Bras Odontopediat Odontol Bebê. 2002;5(24):154-62.

16 Corrêa MSNP, Dissenha RMS, Weffort SYK. Saúde bucal do bebê ao adolescente: guia de orientação. São Paulo: Santos; 2005.

17 Albuquerque SSL, Lima MGGC, Sampaio FC. Avaliação da utilização de dentifrícios fluoretados em pré-escolares na cidade de João Pessoa - Paraíba - Brasil. Odontol ClínCientíf. 2003;2(3):211-6.

18 Brasil. Ministério da Saúde. Secretaria de Atenção à Saúde. Departamento de Atenção Básica. Guia de recomendações para o uso de fluoretos no Brasil. Brasília: Ministério da Saúde; 2009. Disponível em: http://cfo.org.br/wp-content/uploads/2010/02/livro_guia_fluoretos.pdf Acesso em: 02 dezembro 2017.

19 Stuani AS, Stuani AS, Freitas AC, Silva FWFPG, Queiroz AM. Como realizar a higiene bucal em crianças. Pediatria. 2007;2(37):200-7.

20 Cangussu MCT, Narvai PC, Fernandes RC, Djehizian V. A fluorose dentária no Brasil: uma revisão crítica. Cad Saúde Pública. 2002;18(1):7-15.

21 Freddo LP, Aerts DRGC, Abegg C, Davoglio R, Vieira PC, Monteiro L. Hábitos de higiene bucal e utilização de serviços odontológicos em escolares de uma cidade as Região Sul do Brasil. Cad Saúde Pública. 2008;24(9):1991-2000. 
revista de NOVA
ciêncios

da saúdele ESPERANÇA

22. Ramos BC, Maia LC. Cárie tipo mamadeira e a importância da promoção de saúde bucal em crianças de 0 a 4 anos. Rev Odontol Univ São Paulo. 1999;13(3):303-11.

23. Hanna LMO, Nogueira AJS, Honda VYS. Percepção das gestantes sobre a atenção odontológica precoce nos bebês. RGO Rev Gaúcha Odontol. 2007;55(3):271-4.

24. Moraes EM, Valença AMG. Prevalência de gengivite e periodontite em crianças de 3 a 5 anos na cidade de Aracajú. Cienc Odontol Bras. 2003;6(4):87-94. 